\title{
The Meaning of Motions and Their Effects in Einstein's Empty Space and in the Scenario of the Higgs Quantum Fluid Space
}

\author{
Jacob Schaf \\ Physics Institute UFRGS, Porto Alegre-RS, Brazil \\ Email: schaf@if.ufrgs.br
}

How to cite this paper: Schaf, J. (2019) The Meaning of Motions and Their Effects in Einstein's Empty Space and in the Scenario of the Higgs Quantum Fluid Space. Journal of Modern Physics, 10, 256-280. https://doi.org/10.4236/jmp.2019.103018

Received: January 27, 2019

Accepted: March 4, 2019

Published: March 7, 2019

Copyright (อ 2019 by author(s) and Scientific Research Publishing Inc. This work is licensed under the Creative Commons Attribution International License (CC BY 4.0).

http://creativecommons.org/licenses/by/4.0/

\begin{abstract}
Motion is a ground-laying concept in physics. Its meaning however depends fundamentally on the assumptions about the nature of empty space. In Einstein's theory of relativity (TR), no absolute references can be defined and only relative motions are relevant. This however makes it impossible to understand why the motion of matter obeys the principle of inertia and why there exist laws of motion. The Higgs theory introduces radical changes in the current view about the nature of empty space. It introduces the idea that space is filled up by a real and very powerful quantum fluid medium, giving mass to the elementary particles by the Higgs mechanism. This Higgs Quantum Space (HQS) is locally an absolute reference for rest and for motions. It not only recovers an intrinsic meaning for motions, however literally governs the inertial motion of matter-energy. In this new scenario, the velocity of light is fixed with respect to the local HQS and velocity of matter with respect to the local HQS and not relative velocities are responsible for all the effects of motion. The Higgs mechanism is too responsible for the gravitational dynamics; because it is mass that creates the gravitational fields. Actually several clear experimental observations demonstrate that the HQS is moving round the sun consistently with the planetary motions. The present work therefore replaces Einstein's spacetime curvature by a Keplerian velocity field of the HQS. This velocity field creates the ingenious outside-inside centrifuge mechanism of gravity. It also causes all the observed effects of the gravitational fields on light and on clocks.
\end{abstract}

\section{Keywords}

Applied Physics in Gravitation, Gravitational Fields, Gravitational Effects, Gravitation in the Solar System, Higgs Quantum Space in Gravitation 


\section{Introduction}

The first query about the meaning of motions came about from the fact that local mechanical experiments cannot detect uniform rectilinear motion of the laboratory reference, which is known as Galilean invariance of the laws of mechanics. In Einstein's view, the null results of the light anisotropy experiments demonstrate that local electromagnetic experiments cannot reveal the motions of the earth-based laboratories, too. In the Special Theory of Relativity (STR), Einstein generalized this conclusion, postulating that the laws of physics are all invariant with changes of the reference, which he named Principle of Relativity. In order to implement this assumption, he postulated the intrinsic constancy and isotropy of the velocity of light with respect to every possible inertial reference, which, in his view, is confirmed by the null results of the light anisotropy experiments. With base in these facts, Einstein rejected the Aether theories and concluded that empty space contains nothing that can represent a reference for rest and for motions and or be a medium of propagation for light. Motions have no intrinsic meaning. Only relative motions are relevant in physics and only they cause physical effects. In this way, the STR has reduced motions to simple changes in the spatial configuration of reference points as a function of time.

In the absence of a natural reference for rest and for motions, each observer can simply consider him to be hypothetically stationary and define his own proper reference frame, in which stationary clocks show proper time and meter sticks have proper lengths. In Einstein's view, a hypothetical stationary observer can compare the rate of his clocks and the length of his meter sticks with the rate of moving clocks and the length moving meter sticks. He textually writes [1] [2] that, if the velocity of light is isotropic in the reference of the hypothetically stationary observer, it cannot anymore be isotropic, from the view of this stationary observer, in a relatively moving reference. Otherwise, the principle of the constancy of light velocity would be broken. However, anisotropic velocity of light, in the moving reference, is well-known to inexorably lead to an increase of the light go-return round-trip time between two mirrors. In order to make it possible at all that measurements of the velocity of light in the moving reference, by the method of light go-return round-trips and clock, to give the same value as in the hypothetically proper reference, Einstein introduced the idea that, from the view of the stationary observer, clocks in the moving reference run slow in exactly the same proportion as the light go-return round-trip times. Moreover, in order for the moving observer to find light to be isotropic in his reference, Einstein added, apparently motivated by the null results of the $\mathrm{Mi}$ chelson light anisotropy experiments, that distances, in the moving reference, are shortened along the direction of motion exactly in a proportion to make the light round-trips to appear isotropic. From this generic thought experiment, Einstein concluded that relatively moving clocks run slow, moving meter sticks are shortened and postulated that the observed velocity of light is a universal constant $c$ that is intrinsically isotropic in any possible inertial reference. He also 
concluded and postulated that the laws of physics, discovered in one inertial reference, are good in any other inertial reference, which is the Principle of Relativity.

If the observer in a proper reference defines a rectangular Cartesian coordinate frame $(x, y, z)$, and a time axis $t$, the changes in the spacetime coordinates, for relative velocity $V$ along the $x$ axis are described by the famous Lorentz transformations:

$$
\begin{gathered}
x^{\prime}=\frac{x-v_{r} t}{\sqrt{1-v_{r}^{2} / c^{2}}} \\
y^{\prime}=y \\
z^{\prime}=z \\
t^{\prime}=\frac{t-v_{r} x / c^{2}}{\sqrt{1-v_{r}^{2} / c^{2}}}
\end{gathered}
$$

where $c$ is the velocity of light.

Within the scenario of the STR these Lorentz transformations reproduce well the observations on earth. They in particular, predict that a light pulse, emitted from the origins of a stationary and a moving reference frame, at the exact instant their origins coincide, propagates in both references as spherical wave fronts. Observations on earth apparently confirm well the predictions of the STR. However, if they reproduce the experimental observations in other situations never has been tested.

The STR has lead to many fundamental and very important discoveries that actually play part in the human life. However, the characteristic reciprocal symmetry between relatively moving observers leads to many unsolvable paradoxes, for which there are no experimental data to confront. From all the problems with the STR, the most serious shortcoming is the fact that it cannot give a satisfying explanation for the origin of the observed inertial behavior of the matter bodies. In the idle empty space of the STR, it is impossible to understand why matter bodies obey the principle of inertia and why they follow laws of motion. Einstein tried to justify the inertial mass in terms of coupling of local matter to distant matter in the universe, which however entails an arcane instantaneous action from the infinite. A scientifically sound explanation for the origin of the inertial behavior and the inertial mass of matter became known only 60 years later with the Higgs theory. This theory makes radical changes in Einstein's view about the nature of empty space assuming that space is filled up by a real quantum fluid medium.

Actually the atomic clocks in orbit can be synchronized by Einstein's method to within $0.1 \mathrm{~ns}$, time for light to propagate 3 centimeters, which is precision enough to measure the one-way velocity of light. Several experimental observations, achieved with the help of such tightly synchronized atomic clocks in orbit, [3] reveal clear anisotropies of the velocity of electromagnetic signals (light) between the satellites. These observations constitute a conclusive verdict against 
the postulate of the intrinsic isotropy of light. The coming Section 3 gives details. Please see also Refs. [4] [5].

In the General Theory of Relativity (GR) [2] [6] Einstein has introduced the Principle of Equivalence, the equivalence of gravitational and inertial effects. With base in this principle, he extended the ideas of the STR to the physics within gravitational fields. By an ingenious thought experiment he discovered that gravitational fields cause time dilation (slowing of clocks). He imagined an atom in its ground state that after absorbing a photon of energy $E=m c^{2}$, where $m$ is the relativistic mass of the photon, is lifted in the gravitational field by a working force to an altitude $h$. Due to the increased mass of the excited atom, the working force must work harder than for the same atom in its ground state. Thereby the excited atom gains an excess energy of $m g h$. At the higher altitude, the excited atom may relax emitting a photon with the correspondingly increased energy. This increased energy is the excitation energy of this atom at the higher altitude $h$. By this simple thought experiment, Einstein has demonstrated that a gravitational field lowers the atomic frequencies and slows the rate of clocks. For stationary clocks in sufficiently weak gravitational fields, Einstein obtained a gravitational time dilation, given by $t=t_{0}\left(1-2 U / c^{2}\right)^{1 / 2}$, where $2 U=2 G M / r$ is the square of the local escape velocity from the gravitational field, in which $\mathrm{G}$ is the gravitational constant and $\mathrm{M}$ is the mass of the gravitational source. Actually, the atomic clocks, stationary in the earth's field, corroborate very precisely the time dilation predicted by GR.

In order to explain the gravitational dynamics, Einstein has introduced the idea that the mass of large astronomical bodies significantly curve the geometry of spacetime in their neighborhood, causing a mix of space and time components. This curvature is expressed by his famous field equations for the metric tensor $g_{\mu \nu}$ :

$$
G_{\mu \nu} \equiv R_{\mu v}-\frac{1}{2} g_{\mu v} R=8 \pi G T_{\mu v},
$$

In this Equation $R_{\mu v}$ is the Ricci curvature tensor, $R$ is the scalar curvature, $g_{\mu \nu}$ is the metric tensor of the space-time geometry, $G$ is the gravitational constant and $T_{\mu \nu}$ is the mass-energy tensor. In this curved spacetime the path of force-free bodies (in orbit or in free-fall) follows geodesic lines. For weak gravitational fields the curved spacetime is approximately characterized by the invariant length of the line element $\mathrm{d} s$, the square of which, in terms of spherical coordinates $(r, \theta, \phi)$, is given by:

$$
\mathrm{d} s^{2} \approx\left[1-\frac{2 U}{c^{2}}\right]^{-1} \mathrm{~d} r_{0}^{2}+r^{2} \mathrm{~d} w^{2}-c^{2}\left[1-\frac{2 U}{c^{2}}\right] \mathrm{d} t_{0}^{2}
$$

where $\mathrm{w}$ is the angular term. In this equation the coefficients $\left(1-\frac{2 U}{c^{2}}\right)^{-1}$ and $-c^{2}\left(1-2 U / c^{2}\right)$ are respectively the diagonal $g_{11}$ and $g_{44}$ components of the Schwarzschild metric tensor. [7] The last term of this equation expresses the gravitational time dilation as viewed by a stationary external observer, where 
$\mathrm{d} t_{0}$ is an infinitesimal time interval in the absence of a gravitational field. In the first term $\mathrm{d} r_{0}$ is an infinitesimal interval of radial distances in the absence of a gravitational field. According to GR, due to spacetime curvature, the direction of the time axis is different outside and inside a gravitational field. This has the consequence that, from the view of an external observer, the rate of the time evolution of any physical process (clocks) within the field is lowered. The time axis has a space like component, which lets the time like component shorter than $c \mathrm{~d} t_{0}$. Moreover, due to the stretching of the radial distances, in the first term, the velocity of a light pulse, propagating, toward the gravitational center, will appear, from the view of an external observer, to apparently be decreasing. The velocity of light along $r$ has a time like component.

In Einstein's view, the effects of the gravitational acceleration, in a free-falling elevator, are locally canceled. This is often alleged, with base in the principle of equivalence, to recover the situation of a proper reference in which all the effects of the gravitational field are locally canceled. [8] Free-fall certainly cancels locally the gravitational pull and the light bending. However, to present day, no experimental test proves that free-fall cancels the gravitational time dilation in a free-falling reference.

It is well known that, within a gravitational field, any free-falling reference, fulfilling locally the conditions of an inertial reference at a given point of space, is accelerated and non-inertial with respect to free-falling inertial references at all different points of space. References falling along the same radial coordinate recede from each-other at an accelerated rate and references falling along different radial coordinates approximate each-other at an accelerated rate. Therefore, if elevators fall freely from all different possible altitudes, their velocities, at a given spatial point $r_{0}$, have all the possible different velocities from zero up to the local escape velocity $(2 G M / r)^{1 / 2}$. How can all these elevators with all so different velocities, at the same point of space, be proper references? The model of the free-falling references has another even much more serious trouble. It cannot give rise to the gravitational pull. The problem is that the velocity of the free-falling inertial references from the infinite has a constant value $(2 G M / r)^{1 / 2}$ and a fixed direction at any fixed point of space $r_{0}$. A constant velocity with a fixed direction cannot give rise to an inertial pull. Within the context of the TR, obviously none of the free-falling references has an absolute character and none is preferential. From the view of a stationary body at any given fixed point $r_{0}$, the acceleration of the free-falling inertial references is locally zero $\left(\mathrm{d} v / \mathrm{d} t \mid r_{0}=0\right)$. The velocity is a function of $r$ however not of time. Therefore, anybody, brought to rest at any given point $r_{0}$ and then released, will remain stationary in an unstable equilibrium. Any perturbation however will initiate a runaway departure upward or downward.

The next Section 2 introduces the scenario of the Higgs quantum fluid spatial medium, responsible for the mechanical properties of matter. Section 3 shows that a Keplerian velocity field of this Higgs quantum space round the astronomical bodies creates an ingenious outside-inside centrifuge mechanism that gene- 
rates the observed gravitational dynamics and gives rise to all the observed effects of the gravitational fields on light and on clocks. Section 4 implements this centrifuge mechanism in the gravitational dynamics.

\section{The Meaning of Motions in the Scenario of the Higgs Quantum Fluid Space}

The Higgs theory [9] [10] introduces drastic changes in Einstein's view about the nature of empty space. In order to explain the origin of the inertial behavior of the elementary particles, the Higgs theory introduces the idea that space is filled up by a real quantum fluid spatial medium, giving mass and mechanical properties to the elementary particles by the Higgs mechanism. This Higgs Quantum Space (HQS) is much more than simply a reference for rest and for motions. It literally governs the motions of matter-energy. The Higgs mechanism is the perfect HQS analog of the Meissner effect in superconductivity that gives mass to the photons within superconductors [11]. Motion of the elementary particles in the HQS is propagation of confined wave mechanisms that has a well-defined intrinsic meaning. Motion of these wave mechanisms in the HQS involves a lot of physics that gives to the motions an absolutely different meaning than the dynamics of points in the empty space of the STR, in which there is nothing to which the elementary particles can couple and acquire inertial properties.

In the language of the Field Theories, the Higgs quantum fluid spatial medium arises from the breakdown of the electroweak symmetry immediately after the big-bang, caused by the scalar Higgs field, into the weak force doublet and the electromagnetic (EM) field. Subsequently, while the EM field has preserved its $U(1)$ symmetry, the weak-force doublet has spontaneously broken its $U(1)$ symmetry and, by a second order phase transition, has condensed into a macroscopic quantum fluid state, liberating an enormous amount of energy. The condensate has four components, two charged components with spin one, one chargeless component with spin one and one chargeless with spin zero. The three first components are responsible for the mass of the vector bosons

$\left(W^{+}, W^{-}, Z\right)$ by the Higgs mechanism. The forth component, to be referred to by Higgs condensate or Higgs Quantum Space (HQS), remains free and is responsible for the mass of the quarks and leptons by an indirect Yukawa like mechanism. The purpose of the present work is not discussing details of the paraphernalia of the Higgs theory, however to implement its very important impact in the scenario of the universe. Note that without mass there is no linear momentum, no angular momentum and no matter universe.

Likewise usual quantum condensates, the Higgs condensate can be described by the usual macroscopic Ginsburg-Landau [12] like order parameter: $\Phi=\phi(r) \mathrm{e}^{\mathrm{i} \theta}$ where $\phi(r)$ is an amplitude and $\mathrm{e}^{i \theta}$ is a phase factor. In the condensate the phase $\theta$ assumes a well-defined value $\theta_{0}$ between zero and $2 \pi$. This order parameter is a macroscopic wave-function that describes collectively all the bosons of the condensate. It is important to note that, in quantum fluids, no clas- 
sical motions can be considered. In the quantum fluids, the motions (flows) are governed by phase gradients of the order parameter, resulting in velocity fields of the condensate (probability currents). This is not so different from Fermions currents in the atomic orbitals.

The order parameter very strongly suppresses disordered and turbulent motions in the condensate. Moreover, even if the curl of the velocity field $\boldsymbol{V}(\boldsymbol{r})$ of the quantum fluid is non-zero ( $\operatorname{curl} V(\boldsymbol{r}) \neq 0$ ), the flow can be inviscid and totally frictionless (laminar) up to a characteristic limit that depends on each quantum fluid. For instance, in superconductivity, the velocity in the velocity field (screening currents) of the superconducting condensate, confining and quantizing the local magnetic field by the Meissner effect, [13] falls exponentially with the radial distance from the quantized magnetic fluxons. The screening velocity field has a non-zero curl and despite this it is perfectly inviscid, laminar and frictionless. In the case of the HQS the zero viscosity holds up to a limit, given by the $(1 / r)^{1 / 2}$ dependence, where $r$ is the distance from the source.

In terms of the Real and Imaginary components of the order parameter, the characteristic form of the potential well, created in the spontaneous breakdown of the $U(1)$ symmetry of boson systems (superfluids, superconductors and also of the Higgs quantum fluid) has the form:

$$
U(\rho)=-n\left(\Phi^{*} \Phi\right)+m\left(\Phi^{*} \Phi\right)^{2}
$$

Figure 1 visualizes the characteristic form of the potential well of condensed bosons. In Equation (4), the value of the negative coefficient $(-n)$ of the bonding term is considerably larger than the positive coefficient $(+m)$ of the anti-bonding term. Therefore, the minimum of the effective potential energy occurs at a finite value $\Phi^{*} \Phi=n / 2 m$, which is known as a non-zero vacuum expectation value. Many properties of the different quantum condensates are closely analogous. They differ only by the properties of their constituent particles (bosons) and consequently the different fields that couple to them. The Higgs quantum fluid space is referred to as Higgs Quantum Space (HQS), because it forms the space in which the matter universe budges.

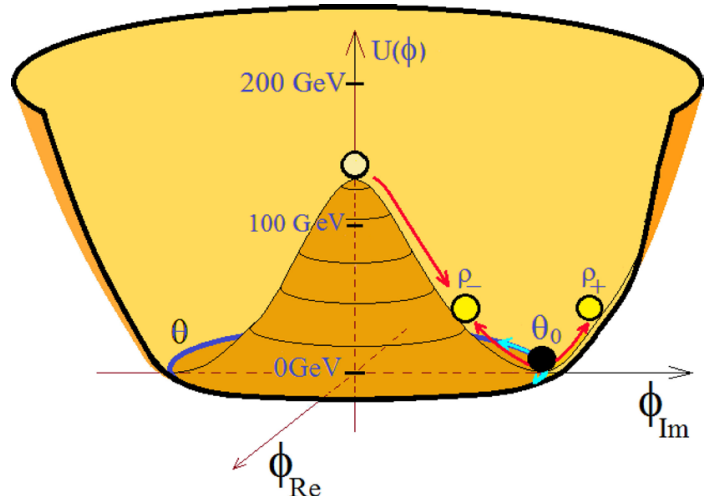

Figure 1. Characteristic potential well of Bose-Einstein Condensates: A red arrow indicates the transition toward the lower energy phase coherent state with the well-defined phase $\theta_{0}$. 
While the conventional quantum fluids (superfluids and superconducting condensates) are stabilized by a low energy gap of only about $1 \mathrm{meV}$ (one millielectronvolt) and condense only at very low temperatures, the transition temperature of the Higgs begins shortly after the big-bang at about $10^{15} \mathrm{~K}$ and the condensate is stabilized by a huge energy gap, that, according to the Glashow-Weinberg-Salam electroweak model, achieves $200 \mathrm{GeV}$. [14] [15] The HQS is an extremely phase rigid and stable quantum fluid. However, likewise the usual quantum fluids, it is at the same time perfectly conservative. Only high energies, in the order of $\mathrm{MeVs}$, can excite local Goldstone modes. However, once excited, these perturbations automatically become indefinitely persistent.

In the conventional and well-known quantum fluids, any local phase gradient causes motion of the condensate along the phase gradient at a velocity proportional to the magnitude of the phase gradient. For instance, an electromotive force difference, applied to the extremities of a superconducting wire, creates an increasing phase gradient along the conductor and an increasing super-current. However, once excited, the current automatically becomes permanently persistent. It can be stopped only by an opposite electromotive force.

Some people insist to call the Higgs quantum fluid spatial medium as quantum aether. In reality this Higgs Quantum Space (HQS) has nothing in common with the Maxwellian aether. The Maxwellian aether is an extremely tenuous hypothetical classical fluid, filling up the empty space. It is at the same time extremely frail and extremely rigid. In GR empty space (vacuum) is described in terms of the stress-energy tensor of a perfect (classical) fluid. A perfect fluid is a system of uncorrelated, well localized and distinguishable ideal particles. The physics of a perfect fluid is a many-body problem. In the Standard Elementary Particle Model, the empty space (vacuum) is described in terms of a nearly infinite number of oscillators. The zero-point energy (vacuum state) of these oscillators leads to the scandalous vacuum energy density that is 120 decimal orders of magnitude larger than shown by observations [14] [15].

The HQS, far from a perfect (classical) fluid, is a macroscopic quantum state of very strongly correlated bosons with spontaneously broken $U(1)$ symmetry and condensed into a highly phase coherent and extremely rigid macroscopic quantum ground state. This condensate is an integrated system, a one body problem, ruled by an extremely powerful Ginsburg-Landau [12] like order parameter. Therefore, the HQS very strongly suppresses the quantum fluctuations and the zero-point energies of the various force fields, turning their contribution to the vacuum energy density irrelevant. The HQS may be conceived as one unique oscillator the size of the universe where the frequency of the Higgs modes tends to zero (takes cosmological eras) and the wave-length tends to the infinite. In this scenario, the universe must be conceived as an adiabatic system, in which the total energy (the condensation energy of the Higgs condensate) is conserved. Actually this energy is composed of the residual condensation energy that still has not been converted into other forms of energy and the energy, due to the ordinary matter. Ordinary matter holds back the advance to the minimum 
energy in the Higgs potential well Equation (4), analogously as a strong magnetic field holds back the superconducting condensate from its advance to the minimum of energy. In the ground state, the amplitude of the Higgs order parameter is intrinsically very closely constant and the density $\rho=\Phi^{*} \Phi=\phi^{2}$ of the condensate is intrinsically highly uniform throughout the volume of the universe, which may solve the horizon and flatness problems of cosmology.

The HQS (Higgs quantum fluid, filling up the whole of space) confines and quantizes the elementary particle fields, giving them inertial mass by a direct or indirect Higgs mechanism. This mechanism is the perfect HQS analog of the Meissner effect in superconductivity. The EM field that couples to the EM vacuum fluctuations does not couple to the HQS and is not confined by the HQS. It remains long-range and the photons have no rest mass in free space. If the HQS gives inertial mass to the elementary particles, it necessarily governs the inertial motion of matter-energy and is the local ultimate, locally absolute and local preferential reference for rest and for motions. The HQS materializes the local Lorentz frames ( $L F S$ ) into local proper LFs, intrinsically stationary with respect to the local HQS. In this scenario, an observer cannot anymore arbitrarily define his proper reference. The local proper references are intrinsically defined by nature, by the local HQS itself. An observer will be in a proper reference only if he is stationary with respect to the local HQS. This will be seen to be very closely the situation of the planets in the solar system and also is the case of the solar system in the Milky-Way galaxy and in the case of galaxies in general throughout the universe. This is the true reason of the isotropy of light with respect to earth and of the universality of the laws of physics [4] [5] [16].

The physical mechanism creating the inertial mass of a field that couples to a quantum condensate is intimately related with the field confining mechanism. In superconductors of type II, the solenoidal vector potential of the magnetic field that couples to the charged superconducting condensate, induces solenoidal phase gradients in the order parameter and, consequently, solenoidal quantized screening currents, the Lorentz forces of which thrust and compress the magnetic field into quantized flux quanta. The inertial behavior of these field quanta (Photons) is due to the fact that their acceleration, within the condensate, needs to create asymmetries in the phase gradients of the order parameter, generating additional screening currents that too are perfectly persistent. This persistence is the origin of their inertial behavior. Quantum fluids have the characteristic property by which any motion and any dynamics, excited in them, is perfectly persistent, giving rise to the inertial behavior. A superconductor also can develop macroscopic screening currents in the border of a superconducting piece. The Lorentz forces, generated by these screening currents, expel the magnetic field (magnetic fluxons) out from the superconductor, because this lowers the effective energy of the superconducting condensate. It also is observed that quickly cooling down a bulky superconductor (from outside), its temperature lowers non-homogeneously and the sample becomes superconducting in the border layer before its interior. In this process the magnetic field is swept inward 
and compressed, letting place behind for additional magnetic flux to penetrate from outside. This is the well-known and intriguing high field paramagnetic Meissner effect of superconductors [17]. The magnetic flux compression in this situation is quite similar to the gravitational compression of the matter fields into astronomical bodies that are bubbles of weakened Higgs order parameter. The different topologies in the Meissner effect and in the Higgs mechanism arise from the different ranking of the confined fields.

Internal circulation of quantum fluids can in no way be conceived as classical motions. In quantum physics even the motion of individual particles must be conceived in terms of the propagation of modulated wave-packets. The motion of such wave-packets or wave-functions has locally a wave character; however it too has classical aspects in the measure the wave-packet is localized in a finite region of space, which is the particle character. This is the wave-particle duality. Note that even a superconductor, if it is localized within a finite region of space, has classical aspects. For instance, a cold superconducting piece can move like a classical body. However, the volume of the HQS visibly has no limiting border, which eliminates totally the possibility of observing classical aspects in it. In quantum fluids the wave functions of the bosons are fully entangled. They have lost their individuality and are indistinguishable. The phases of the wave-functions of individual bosons are coherent with those of all the other bosons of the condensate. They are extended and have non-zero amplitude throughout the volume of the condensate. Within the quantum fluid there is nothing like a succession of positions of its parts as a function of time. Likewise the motion of electrons in atomic orbitals, the circulation in quantum fluids (probability currents) are created and governed by phase gradients of the order parameter and internally exhibit no classical aspects. Although perfectly real, the flow of quantum fluids is almost totally imperceptible. Only very specific fields, coupling to the quantum fluid are affected by this motion. If the circulation within the quantum fluid is along a closed loop, (analogously as in the atomic orbitals), the phase factor necessarily satisfies quantization rules. This is well known in superfluids and in superconductors and gives rise to the quasi-particles: Rotons, Maxons and Vortices.

If the Higgs mechanism creates the inertial mass of matter, it necessarily is responsible too for the gravitational dynamics, because it is mass that creates the gravitational fields. Without mass there are no gravitational fields and no gravitational dynamics. Likewise the usual quantum fluids move along phase gradients of the respective order parameter, the Higgs quantum fluid medium, too can itself move, in the ordinary space, along phase gradients according to a well-defined velocity field. If this velocity field has a velocity gradient, it creates inertial dynamics on matter-energy, which according Einstein's principle of equivalence is gravitational dynamics. Actually, several experimental observations (please see the next Section 3), indicate that the HQS is moving round the sun according to a velocity field consistent with the orbital motion of earth. In such a velocity field earth is stationary with respect to the local HQS and is very 
closely a proper reference (proper Lorentz frame) in which clocks show proper time and light is isotropic.

The Higgs Quantum Space (HQS) is much more than simply a local ultimate (locally absolute) reference for rest and for motions. It literally governs the inertial motion of matter-energy (wave-packets). In the scenario of the HQS, the velocity of light has a fixed value $c$ and is isotropic with respect to the local HQS and not with respect to every possible observer. This velocity $c$ is the maximum velocity at which the order parameter of the Higgs condensate (HQS) transmits the phase perturbations. The HQS provides a locally absolute meaning to the motions. On the other hand, the velocity of light is anisotropic in any reference (LF) moving with respect to the local HQS. Moreover, all effects of motion on particles, on light and on clocks are due exclusively and uniquely to velocity with respect to the local HQS and not to relative velocity. The local HQS constitutes a local absolute reference for rest and for motions and the velocity with respect to the local HQS is absolute velocity, however only locally.

In the TR, the elementary particles are seen as existences in themselves, innately having mass, charge, spin etc. They are intrinsically associated with fields. According to this theory, their motion has no intrinsic meaning and only relative velocity is relevant to physics. In the Higgs theory the elementary particles are seen as confined field (wave) objects, as local perturbations in the Higgs order parameter. Their motion with respect to the local HQS has yes locally an intrinsic meaning. The HQS offers no mechanical resistance to the motion of these particles because; 1) it is a perfectly conservative quantum fluid and 2) these particles propagate in it as localized wave mechanisms and hence cannot collide with the local HQS. Likewise usual water and sound waves, these wave mechanisms, from the perspective of an observer moving with respect to the local HQS, are affected by Doppler shifts. However, likewise usual quantum fluids the HQS too can itself move according to a certain velocity field and distort it causing wavelength stretching-contraction and refractions. Actually various experimental observations demonstrate that the HQS is moving round the sun and round earth according to macroscopic Keplerian velocity fields, giving rise to the ingenious outside-inside centrifuge mechanism of gravity that is responsible for the observed gravitational dynamics.

\section{The Effects of Motion with Respect to the Local HQS in Free-Space and within Gravitational Fields}

In conventional physics, motion is change of position in the ordinary space as a function of time. In order to describe motions, it is necessary to define first a reference frame with space and time coordinates. A system of orthogonal Cartesian axes $(x, y, z)$, scaled by units of length and a clock scaled by units of time $t$ is the most usual definition. The position and motion of a point can be made by specifying the coordinates along the $x, y$ and $z$ axes as a function of time $t$, or in terms of the spherical coordinates $(r, \theta, \phi)$ as a function of time $t$. If this refer- 
ence frame represents the absolute rest, velocities with respect to it are absolute velocities. In his Theory of Relativity (TR) Einstein rejected the possibility of an absolute reference and hence, from the view of the TR, absolute motions are a meaningless concept. Einstein insisted that only relative motions and relative velocities are relevant in physics. In this scenario, every observer can arbitrarily define his own proper reference and consider all the other references as moving references. However, all the proper references, defined by all the different observers are equivalent and none of them is preferable. This leads to the problematic reciprocal symmetry between inertial observers. Consider two observers A and $B$ in motion with respect to each other. In the view of each observer, his clock shows proper time and his meter sticks have proper lengths. However, in the view of observer $A$, the clock of $B$ runs slow and in the view of observer $B$, the clock of A runs slow. This is the famous twin paradox to which there is no possible solution. Many other unsolvable paradoxes can be found in the literature.

In the scenario of the HQS, the definition of references is radically different from that in the TR. In the scenario of the HQS, which is the local ultimate (locally absolute) reference for rest and for motion of matter-energy, Einstein's inertial observer cannot anymore arbitrarily define his own proper reference. He only can consider him to be in a proper reference if he is stationary with respect to the local HQS. The Local HQS is the local proper (absolute) reference for rest and for motion. However, the HQS can itself move and, if this motion is non-homogeneous, the reference changes from point to point. Therefore, a universal absolute and proper reference is not possible. Only local absolute references can be defined, in which a stationary clock shows proper time and light is isotropic. Any local reference, moving with respect to the local HQS is not a proper reference. In this scenario, the references of two observers, at different points of space, can both be proper references. This however is possible only if both are stationary with respect to the local HQS. It will be seen that the planets of the solar system are all very closely stationary with respect to the local HQS and so implement very closely this situation.

Clocks count time with the help of a time standard that may be a classical or a quantum oscillator. Such oscillators are essentially wave-packets propagating in go-return round-trips within a potential well. In the case of the atomic clocks, the time standard is an electromagnetic (EM) cavity in which the oscillations of the EM field are tuned to the very stable frequency of the hyperfine transition of Cs atoms. These clocks can measure time within a precision better than $0.1 \mathrm{~ns}$. However, such time standards are affected by velocity of the clocks with respect to the local HQS. The oscillation period of the time standard is ruled by the effective velocity of the wave-packets (Photons) in the go-return propagation within the cavity and within the atomic shells of the Cs atoms. Such effects are well-known from the Ives-Stilwell like experiments [18]. It however is important to note that in these experiments the Hydrogen atoms speeded at several thou- 
sand $\mathrm{km} / \mathrm{sec}$. The experimental observations, to be discussed here, after the coming Equation (6) below, show that the earth-based laboratories do not achieve $8 \mathrm{~km} / \mathrm{sec}$ ), which certainly cannot introduce a detectable effect in the Ives-Stilwell experiments. The period $T$ of the round-trip times of the time standards, moving at a velocity $v$ with respect to the local HQS, is given by the well known equation:

$$
T(v)=T_{0}\left(1-v^{2} / c^{2}\right)^{-n}
$$

where $T_{0}$ is the period of a Cs atom stationary with respect to the local HQS (the proper reference). The exponent is $n=1 / 2$ for oscillations transverse to $v$ and $n=1$ for longitudinal oscillations. Hence, the clock rate depends on the value of the velocity $v$ of the clock with respect to the local HQS and also on the direction of the oscillations of the time standard with respect to the velocity vector of the clock. Interestingly, the rate of clocks decreases in exactly the same proportion as the light go-return round-trip time between two mirrors. This is not a mere coincidence. In fact, the go-return light round-trips between two mirrors and the go-return of the electromagnetic field in the potential well of the time standard (oscillator) are affected in exactly the same proportion by motion with respect to the local HQS [18]. Therefore, measurements of the velocity of light, by the method of light go-return round-trips and clock, necessarily are independent from the velocity of the laboratory with respect to the local HQS. However, this invariance clearly is an experimental artifact and in no way proves that the one-way velocity of light really is an invariant.

Actually, several clear-cut experimental observations are in conflict with the relativistic conception. Clocks stationary within gravitational fields (on earth) show exactly the gravitational slowing predicted by GR (please see last term in Equation (3)). The GPS clocks, moving with earth round the sun, could easily detect the gravitational slowing by the solar field. They however show no any effect [19] [20]. Clearly the orbital velocity of earth cancels the gravitational slowing by the solar field, demonstrating that earth effectively is a proper reference (stationary with respect to the local HQS). Current theories explain this absence in terms of Einstein's principle of equivalence. However, these same GPS clocks too are moving along circular orbits round earth and, according to this interpretation of the principle of equivalence, this orbital motion too should cancel the gravitational slowing be the earth's field. However, the gravitational slowing by the earth's field is well observed. References [4] [16] explain exactly why.

In the past century a large number of light anisotropy experiments, performed on earth, searching for light anisotropy, due to the orbital and cosmic motion of earth, gave null results. From the perspective of the present HQS, these observations consistently confirm that earth is stationary with respect to the local HQS and is a proper reference (a proper Lorentz frame). The absence of the gravitational slowing of the GPS clocks by the solar field and the absence of light anisotropy with respect to the moving earth, in reality are the signature of the true 
physical mechanism of gravity, as will be shown hereafter. Einstein could have concluded from the observed null results of the light anisotropy experiments that earth is stationary with respect to the spatial medium propagating light. Rapidly he would have discovered the ingenious outside-inside centrifuge mechanism of gravity [4] [16]. He nevertheless had reasons to think otherwise.

Actually the atomic clocks in orbit can be synchronized by Einstein's method to within a precision of 0.1 ns or better, time for light to travel 3 centimeters. For clocks moving in polar orbits, this synchronization method is especially favorable, because the go-return travel time between the earth-based station and the satellites is isotropic along the whole orbit (please see Equation (6) below). With the help of these tightly synchronized atomic clocks, the one-way velocity of electromagnetic signals (light) has precisely been measured. Such measurements are very helpful to define the characteristic form of the velocity field of the HQS. The most precise measurements of the one-way velocity of EM signals (light) was achieved with the help of the atomic clocks in the robotic twin satellites of the GRACE project, moving at nearly $8 \mathrm{~km} / \mathrm{sec}$ along a same polar orbit, at 500 $\mathrm{km}$ of altitude and separated by about $200 \mathrm{~km}$. In the measurements of microgravity effects, EM signals are continuously exchanged forward and backward between these satellites. Moreover, their clocks need to be synchronized to better than $0.16 \mathrm{~ns}$. In these experiments, the one-way velocity of the EM signals was measured in both senses. The results have shown a clear anisotropy of about 8 $\mathrm{km} / \mathrm{sec}$, backward to the orbital motion of the satellites. This value is exactly the orbital velocity of the satellites and corresponds to about $17 \mathrm{~ns}$ of excess or shortage of time of flight [3]. This observation unambiguously breaks the intrinsic isotropy of light and has the consequence that the null light anisotropy results, found in the past century, need a new interpretation. In fact the observed null results of the light anisotropy experiments are not due to the intrinsic isotropy of light, however to the fact that the moving earth is stationary with respect to the local HQS.

The above experimental observations all together demonstrate that earth is stationary with respect to the local HQS in the solar field and that the velocity of the HQS round earth (and round the sun) has no North-South component. However, earth obviously cannot be considered to be in a privileged kinematical condition in detriment to all the other planets. All the planets must equally be nearly stationary with respect to the local HQS. This is not difficult to accomplish as the planets move all along direct circular orbits that lie all closely within the plane of the solar system. The above described experimental observations can make a sense only if the HQS is circulating round the sun according to a Keplerian velocity field, consistent with the planetary orbital motions and round earth consistently with the orbital motion of the Moon. In spherical coordinates, the form of the velocity field $V(r, \theta, \phi)$ is:

$$
\boldsymbol{V}(r)=(G M / r)^{1 / 2} \boldsymbol{e}_{\phi}
$$

In this Equation, $G$ is the gravitational constant, $M$ is the mass of the gravita- 
tional source (sun), $r$ is the radial spherical coordinate and $\boldsymbol{e}_{\phi}$ is a unit vector along the azimuthal spherical coordinate $+\phi$. This is a very simple velocity field round the $Z$ axis. It has a non-null component only along the $+\phi$ spherical coordinate. In this velocity field the magnitude of the velocity is spherically symmetric. This Keplerian velocity field is responsible for the ingenious outside-inside centrifuge mechanism of gravity that is the quintessence of the gravitational fields. Section 4 will show that it accurately creates the observed gravitational dynamics, the orbital motions, the gravitational acceleration, the gravitational pull etc. Refs. [4] [5] [16] also show that it accurately produces all the observed effects of the gravitational fields on light and on clocks. It causes the observed gravitational slowing of the atomic clocks, stationary in a gravitational field, exactly as predicted by the GR. It predicts the absence of the gravitational slowing of the GPS clocks by the solar field. [4] It predicts the absence of light anisotropy with respect to earth. It precisely predicts the observed Shapiro effect [21] not as a result of the increased geometrical distances, due to the spacetime curvature, however as a result of the effective velocity of the radar signals ( $\left.c^{\prime}=c \pm G M / r\right)$ in the the go and return travels in the solar Keplerian velocity field of the HQS. [4] [16] It also predicts all the other observed effects of the solar and the earth's gravitational fields on light and on clocks. Sections 4 gives more details.

In the solar Keplerian velocity field Equation (6) that achieves $436 \mathrm{~km} / \mathrm{sec}$ on the solar surface and about $30 \mathrm{~km} / \mathrm{sec}$ at the earth's orbit, earth is moving round the sun along a direct nearly circular equatorial orbit at a velocity very closely equal to that of the local HQS. The absence of the gravitational slowing of the GPS clocks by the solar field and the absence of light anisotropy, with respect to earth, show that earth effectively is very nearly stationary with respect to the local moving HQS. It is locally very closely a true proper and preferential reference, a local proper Lorentz frame. The velocity of light is isotropic with respect to earth, not because of the intrinsic isotropy of light, however because the velocity of light is fixed with respect to the local HQS and earth is stationary with respect to this locally moving HQS.

Earth has its own Keplerian velocity field of the HQS in the sense of the Moon's orbital motion (toward the East). The earth's velocity field achieves 7.91 $\mathrm{km} / \mathrm{sec}$ on surface. However, earth rotates only very slowly. Therefore, the earth-based laboratories have a velocity with respect to the local HQS of nearly $V=8 \mathrm{~km} / \mathrm{sec}$, toward the West and are nearly, however not exactly proper references. The effect of this velocity on light and on clocks is extremely small and constant the whole day and the whole year, in the order of $V^{2} / c^{2}=10^{-10}$ and very difficult to detect by light anisotropy. Light anisotropy of about $8 \mathrm{~km} / \mathrm{sec}$ has been detected by only some of the most sensitive genuine Michelson experiments [22]. Please see Figure 2.

The effects of this small velocity however are well detected by the gravitational slowing of atomic clocks on earth. [8] It also has been detected by Mössbauer effect in the atomic spectral red-shifts [23]. The HQS gravitational mechanism 


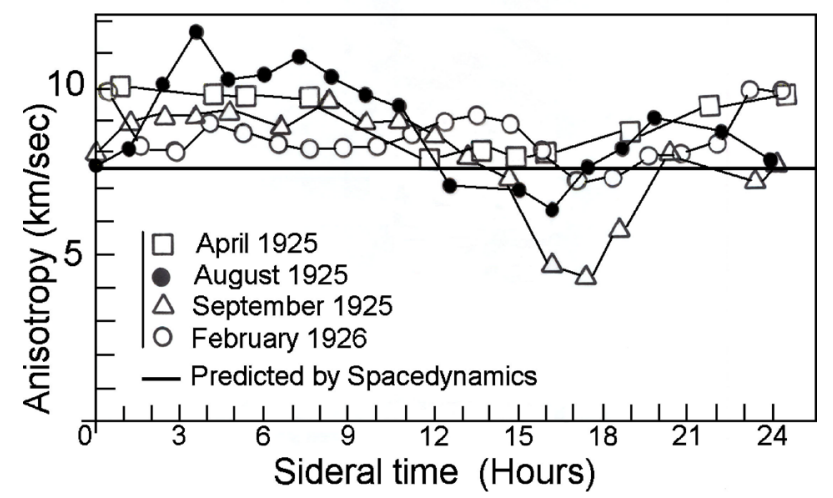

Figure 2. The Nearly West-East anisotropy of light, with respect to the earth-based laboratories, constant the whole day and the whole year, by D. Miller.

too gives a simple explanation to the gravitational slowing of the GPS clocks by the earth field, that are moving round earth along non-equatorial orbits, making $55 \mathrm{deg}$ with the earth's equator [4]. It also predicts dragging of the Neutrinos along their path from CERN to Gran-Sasso IT Southeast, making 58 deg with the Meridians. The West-East velocity of the HQS in the earth's Keplerian velocity field boosts up the velocity of Neutrinos as well as of light by $6.7 \mathrm{~km} / \mathrm{sec}$, causing them both to arrive $55 \mathrm{~ns}$ too early to Gran-Sasso. However, this too early arrival of the Neutrinos in no way proves that Neutrinos exceed the light velocity. In the mega-experiment, to be implemented in coming years, Neutrinos will be shot toward the West from Fermilab (IL) to Stanford (SD). In this case, the Neutrinos are predicted to reach Stanford too late by about 115 ns. Please see details in Ref. [24].

The theory of relativity (TR) has lead to a number of very important discoveries, like time dilation and the increase of mass with velocity. It provided the relation between mass and energy and has lead to the discovery of the gravitational time dilation etc. Actually, many experimental observations, realized in the earth-based laboratories, with particles speeding at very high velocities (comparable to the velocity of light), apparently corroborate the predictions of the TR. However, from the present HQS dynamics view, earth is very closely stationary with respect to the local HQS in the Keplerian velocity field of the HQS, creating the solar field. Moreover, the solar system is stationary in the Milky-Way galaxy etc. Earth is very closely a true and not a hypothetical proper reference. However, because of the bold assumption of the TR that the earth-based laboratories can be assumed by the laboratory observers to be hypothetically stationary references, the predictions of the TR, for experiments on earth, coincide very closely with the predictions in the scenario of the HQS dynamics. This coincidence however is true only in this specific and very particular circumstance. In other circumstances this coincidence is predicted to fail. In reality these experimental observations in no way corroborate the TR. They in fact simply show the effects of the high velocity with respect to the local HQS, in which the earth-based laboratories are nearly stationary. Earth is very closely a true proper reference. Saying 
that these observations corroborate the TR is like assuming that the atmospheric pressure is a universal constant throughout the universe and claiming that precise measurements (of the atmospheric pressure) in the hold of a ship, navigating round earth, confirm this naif assumption.

In free space, the HQS is the local ultimate (local absolute) reference for rest and for motions of matter-energy. However, within gravitational fields, the HQS moves according to a Keplerian velocity field, given by (Equation (6) and can only locally be a proper (absolute) reference. Moreover, such local proper references are themselves moving and rotating round an over-head axis with the local HQS in the Keplerian velocity field. Therefore, within gravitational fields, it is impossible to define a universal proper reference in the sense of the conventional view. Only local proper references can be defined, which themselves are moving with the local HQS. In this scenario, the planets in the solar system that are moving with the local HQS along different orbits within the plane of the solar system, are each one locally very closely stationary with respect to the local moving HQS and are each one locally nearly a proper reference. Their slightly elliptic orbits give account that they have small velocities, with respect to the local HQS, only of hundreds of $\mathrm{m} / \mathrm{sec}$. Each planet is locally very closely (however not exactly) a proper reference, with respect to which the velocity of light is very nearly isotropic and clocks, moving with them, show very closely proper time.

The velocity of the planets can be measured from earth, using the spectral frequency shifts. In reality the planets are locally very closely stationary with respect to the local HQS and locally emit the radiation with proper frequency. These frequencies however are shifted by the time rate of stretching compression of the wavelengths by the velocity field of the HQS along the path of light, simulating Doppler shifts. It can be shown that the values of these frequency shifts are identical to the Doppler shifts, caused by their conventional relative velocities. The frequency of light, coming from the stars in the distant galaxies is shifted by the local wavelength stretching-compression within the velocity field of the local HQS, generating the respective galactic gravitational dynamics as well as by the cosmic expansion of the HQS, causing the recession between the galaxies. The isotropy of light, with respect to earth, is the most far-reaching evidence that earth is very nearly stationary with respect to the local HQS and is closely a proper reference. Visibly, the astronomical bodies throughout the universe are all very nearly stationary with respect to the local HQS in the respective gravitational fields and are nearly proper references. This entails the universality of the laws of physics without the need of the Principle of Relativity.

\section{Origin of the Gravitational Dynamics in the Keplerian Velocity Field of the HQS}

The present work associates the central idea of the Higgs theory, according to 
which the HQS governs the inertial motion of matter, with the central idea of GR, according to which the gravitational dynamics is inertial dynamics and replaces Einstein's spacetime curvature by a Keplerian velocity field Equation (6) of the HQS. In this Keplerian velocity field, the horizontal velocity of the HQS along the $+\phi$ increases with decreasing radial coordinates $r$ in the neighborhood of any point of space. This creates a rotation of the local HQS and thus of the local inertial references round an over-head axis. The idea that the local rotating HQS represents locally a rotating inertial reference may seem stupid. In fact however it is not stupid at all, because it is the HQS, ruling the inertial motion of matter-energy and is the local ultimate (proper) reference for rest and for motions that is itself locally so rotating. Hence, a body, stationary in the ordinary space within a gravitational field (within the Keplerian velocity field), is implicitly moving oppositely along a circular path, within de locally proper inertial reference, round the same over-head axis and necessarily under an upward centripetal force. In this scenario, the gravitational pull essentially is a centrifugal pull toward the gravitational center. This gives rise to the ingenious outside-inside centrifuge mechanism of gravity that Einstein has missed. It is a completely new and physically genuine gravitational mechanism that continuously pulls us against the ground. This ingenious outside-inside centrifuge mechanism resolves one of the most recurrent conundrums of fundamental physics. It fully and transparently elucidates the origin of the gravitational pull and of the gravitational dynamics. This Keplerian velocity field of the HQS is consistent with the planetary orbital motions and is the quintessence of the gravitational fields. It accurately creates the observed gravitational dynamics without the need of a central force field and also correctly generates all the effects of the gravitational fields on light and on clocks. A detailed description of all these effects is here impossible because it would take too many pages. The interested reader is advised to Refs. [4] [5] [16] were a detailed description is given. Here only some basic steps will be outlined.

The vorticity of the HQS in the Keplerian velocity field Equation (6) is intermediary between rigid-body rotation and the irrotational potential flow. Due to the increasing horizontal velocity of the local HQS with decreasing $r$ in this velocity field, the wave fronts of the matter waves of the particles, stationary in the ordinary space and thus moving with respect to the local HQS along $-\phi$ (wave fronts in the $[r, \theta]$ plane) are refracted at a locally well-defined time rate. Moreover, due to the characteristic Keplerian velocity field, the $\phi$ and the $r$ velocity components are refracted in opposite senses. While the $\phi$ component is refracted, due to the increasing (horizontal) velocity of the HQS along $+\phi$ for decreasing radial coordinate, giving rise to the rotation of the local inertial reference round the over-head axis, the $r$ velocity component (wave-fronts in the $(\theta, \phi)$ plane is refracted oppositely, due to the variation of the local velocity of the HQS round the gravitational center with decreasing $r$. The rotation of the $r$ component is a residual effect, reminiscent from the rigid-body rotation round 
the gravitational center. The $\theta$ velocity component is not refracted at all, because the velocity of the HQS in the Keplerian velocity field Equation (6) has no component along the $\theta$ coordinate and becuse the velocity along $\phi$ is constant with $\theta$ (for fixed $r$ ). The opposite refraction rates of the $r$ and the $\phi$ velocity components constitutes not a trigonometric however a hyperbolic rotation. Figure 3 displays velocity diagrams along an elliptic orbit round the gravitational source with eccentricity $\epsilon=0.5$. The diagrams show the precisely calculated orbital velocities $v_{o r b}$ of the orbiting particle, the velocity of the local HQS and the effective velocity $v_{e f f}$ of the particle with respect to the local HQS at a large number of positions along the orbit.

In this figure, the effective rotation rates of the $\phi$ velocity component (top) and of the $r$ velocity component (left) can precisely be read. The effective rotation rates $\boldsymbol{W}$ of the $r, \phi$ and $\theta$ velocity components are given by the equations:

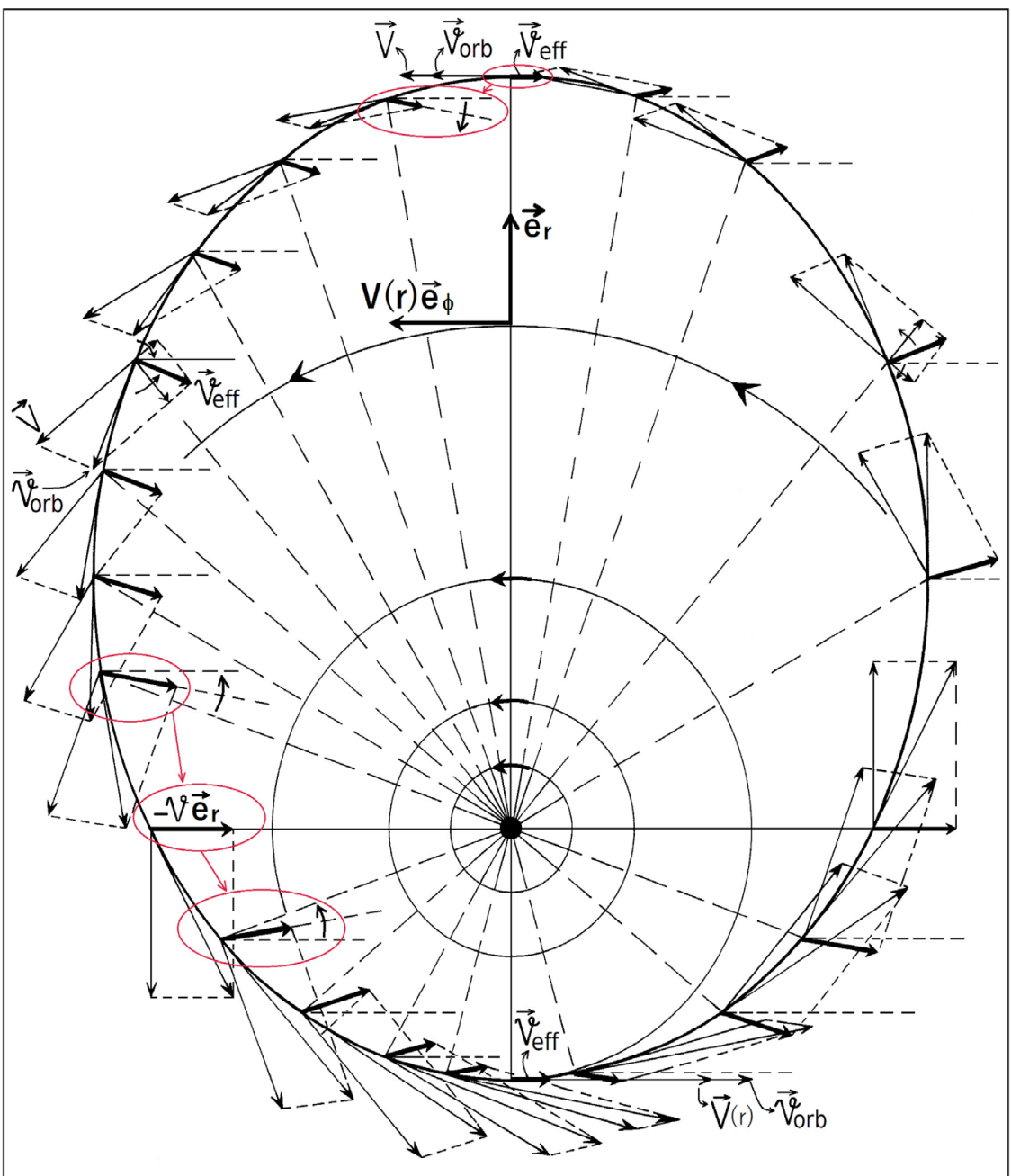

Figure 3. The figure is a very precise graphical representation, showing the velocity diagrams at a large number of positions along the orbit, where $\boldsymbol{v}_{\text {eff }}$ (bulky arrows,) was obtained using the vector relation: $\boldsymbol{v}_{\text {eff }}+\boldsymbol{V}(r)=\boldsymbol{v}_{\text {orb }}$. 


$$
\begin{gathered}
\boldsymbol{W}_{r}(r)=-\frac{1}{2}\left[G M / r^{3}\right]^{1 / 2} \boldsymbol{e}_{\theta} \\
\boldsymbol{W}_{\phi}(r)=+\left[G M / r^{3}\right]^{1 / 2} \boldsymbol{e}_{\theta} \\
\boldsymbol{W}_{\theta}(r)=0
\end{gathered}
$$

These rotation rates depend only on the radial coordinate $r$, because the velocity gradient of the velocity field Equation (6) has only a radial component. These effective rotation rates include the effects of the wavelength stretching-compression. Note that these opposite rotation rates that are different for $r$ and $\phi$ components characterize a non-symmetric hyperbolic rotation. This is the characteristic that naturally accomplishes the Virial theorem.

A particle of mass $m$, stationary in the ordinary space, within the Keplerian velocity field, created by a mass $M$, has an implicit velocity (wave fronts in the $[r, \theta]$ plane) pointing along $-\phi$ :

$$
\boldsymbol{V}_{\text {impl }}=-(G M / r)^{1 / 2} \boldsymbol{e}_{\phi}
$$

This velocity is implicit because it cannot be described in the ordinary space. It is the negative of Equation (6). The refraction rate of this implicit velocity, according to Equation (7b), generates an instantaneous ordinary vertical downward acceleration given by:

$$
\boldsymbol{g}(r)=W_{\phi}(r) \boldsymbol{e}_{\theta} \times V_{\text {impl }}(r)\left(-\boldsymbol{e}_{\phi}\right)=-G M / r^{2} \boldsymbol{e}_{r}
$$

This expression is exactly the usual expression for centrifugal accelerations in a rotating reference. It describes a central field of centrifugal accelerations toward the gravitational center from the equator to the Poles. However, within the Keplerian velocity field Equation (6), the local non-inertial rotating reference, in which the particle is stationary, does not rotate in the ordinary space. Its rotation is implicit and opposite to the ordinary rotation of the local proper inertial reference. These opposite rotations cancel in the ordinary space.

The planets in the solar system are all moving very closely along circular orbits that lie all closely within the equatorial plane of the solar Keplerian velocity field (Equation (6)). This minimizes their velocity and kinetic energy with respect to the local HQS. The velocity of the planets with respect to the local moving HQS is very low, only of a few hundreds of $\mathrm{m} / \mathrm{sec}$. The planets thus are very closely proper Lorentz frames, in which all the effects of the solar field are locally very nearly canceled. Therefore, clocks, moving with the planets, show proper time, the velocity of light is isotropic with respect to them and the gravitational pull of the solar field on them is locally zero. All this is valid too for the Moon, which too is moving along a closely direct circular equatorial orbit round earth in approximately the equatorial plane of the earth's Keplerian velocity field. The gravitational tides are residual effects, due to the mutual distortions in the respective Keplerian velocity fields of the HQS.

The expression Equation (9) is precise enough only as long as the vertical velocity is very low. In order to get a precise description for free fall along large 
radial distances, it is necessary to solve the elementary linear differential equation:

$$
\frac{\mathrm{d} \boldsymbol{v}(r(t))}{\mathrm{d} t}=\boldsymbol{A} \boldsymbol{v}_{0}
$$

where $v_{0}$ and $v$ are respectively the initial and the final column velocity matrices of the (ordinary) $r$ and the (implicit) $\phi$ velocity components of the effective velocity with respect to the local HQS:

$$
\boldsymbol{v}(t)=\left(\begin{array}{l}
v_{r}(t) \\
v_{\phi}(t)
\end{array}\right)
$$

and $\boldsymbol{A}$ is the hyperbolic rotation matrix (a rank 2 tensor) defined in terms of the rotation rates, given in Equation (7) as:

$$
\boldsymbol{A}=\left(\begin{array}{cc}
0 & W_{\phi} \\
-W_{r} & 0
\end{array}\right)=\left(\begin{array}{cc}
0 & W \\
\frac{1}{u} W & 0
\end{array}\right)
$$

where $W=G M / r^{3}$ and the coefficient $u=2 M /(M+m)$ accounts for the asymmetric distribution of kinetic energies between the interacting masses $m$ and $M$, that is approximately 2 for $m \ll M$ and is 1 for $m=M$. This matrix effectuates hyperbolic rotations round parallel axes of the velocity components in Equation (11).

Dividing both sides of Equation (10) by $\boldsymbol{v}_{0}$, multiplying them by $\mathrm{d} t$ and integrating the left hand side from $v_{0}$ to $v$, develops into:

$$
\log \frac{\boldsymbol{v}(t)}{\boldsymbol{v}_{0}}=\int_{0}^{t} A\left(r\left(t^{\prime}\right)\right) \mathrm{d} t^{\prime}
$$

where $A \mathrm{~d} t$ is an infinitesimal rotation round parallel axes. These successive infinitesimal rotations commute. Equation (13) can be re-written in the exponential form as:

$$
\boldsymbol{v}(t)=\exp \left[\int_{0}^{t} A\left(r\left(t^{\prime}\right)\right) \mathrm{d} t^{\prime}\right] \boldsymbol{v}_{0}
$$

Expanding the exponential in series and adding up the terms of the series from $n=0$ to $n=\infty$ results in:

$$
\begin{aligned}
\boldsymbol{v}(t) & =\sum_{n=0}^{\infty} \frac{1}{n !}\left(\begin{array}{cc}
0 & \Theta(t) \\
\frac{\Theta(t)}{u} & 0
\end{array}\right)^{n}\left(\begin{array}{l}
v_{r}(0) \\
v_{\phi}(0)
\end{array}\right) \\
& =\left(\begin{array}{cc}
\cosh \left(\frac{\Theta(t)}{\sqrt{u}}\right) & \sqrt{u} \sinh \left(\frac{\Theta(t)}{\sqrt{u}}\right) \\
\frac{1}{\sqrt{u}} \sinh \left(\frac{\Theta(t)}{\sqrt{u}}\right) & \cosh \left(\frac{\Theta(t)}{\sqrt{u}}\right)
\end{array}\right) \times\left(\begin{array}{l}
v_{r}(0) \\
v_{\phi}(0)
\end{array}\right)
\end{aligned}
$$

which is the general solution of Equation (10).

The value of $\Theta(t)$ can be computed by integration, making use of the change of variable $\mathrm{d} t=>\mathrm{d} r$ method. Inverting the obtained result, gives: 


$$
\begin{gathered}
\cosh \left(\frac{\Theta(t)}{\sqrt{u}}\right)=\sqrt{\frac{r_{0}^{\mathrm{CM}}}{r^{\mathrm{CM}}}}=\sqrt{\frac{r_{0}}{r}} \\
\sinh \left(\frac{\Theta(t)}{\sqrt{u}}\right)=\sqrt{\frac{r_{0}^{\mathrm{CM}}-r^{\mathrm{CM}}}{r^{\mathrm{CM}}}}=\sqrt{\frac{r_{0}-r}{r}}
\end{gathered}
$$

where $r_{0}$ is the initial radial coordinate. Using this result in Equation (15) the general solution takes the form:

$$
\left(\begin{array}{l}
v_{r}(t) \\
v_{\phi}(t)
\end{array}\right)=\left(\begin{array}{cc}
\sqrt{r_{0} / r} & \sqrt{u\left(r_{0}-r\right) / r} \\
\sqrt{\left(r_{0}-r\right) / u r} & \sqrt{r_{0} / r}
\end{array}\right) \times\left(\begin{array}{l}
v_{r}(0) \\
v_{\phi}(0)
\end{array}\right)
$$

and the particular solution of Equation (10) for free fall of $m$ in the field of $M$ $(m \ll M)$, on from initial rest at $r_{0}$, where $v_{r}(t=0)=0$ and $\boldsymbol{v}_{\phi}(0)=\boldsymbol{V}_{\text {impl }}\left(r_{0}\right)=-\left(G M / r_{0}\right)^{1 / 2} \boldsymbol{e}_{\phi}$ is:

$$
\begin{gathered}
v_{r}(t) \approx-\left[2\left(\frac{G M}{r(t)}-\frac{G M}{r_{0}}\right)\right]^{1 / 2} \boldsymbol{e}_{r} \\
v_{\phi}(t)=-V_{\text {impl }}\left(r_{0}\right)\left[\frac{r_{0}}{r}\right]^{1 / 2}=-\left[\frac{G M}{r(t)}\right]^{1 / 2} \boldsymbol{e}_{\phi}
\end{gathered}
$$

Equation (18a) is just the well known expression for the observed vertical free-fall velocity on from rest at $r_{0}$. It directly shows that the kinetic energy $1 / 2 m v_{r}^{2}$ is equal to the difference between the final and the initial potential energies. Equation (18b) is just the implicit (imaginary) velocity as a function of the radial position $r$. It shows that the refraction rate of the radial velocity component just compensates for the increase of the velocity field as a function of the decrease of the radial coordinate. This assures that free-fall of the particle, on from rest, goes along a vertical (radial) path and hence assures conservation of the angular momentum about the gravitational center. Please observe that, for free-fall on from infinity $\left(r_{0}=\infty\right)$, the vertical velocity $v_{r}(r(t))$ is exactly $\sqrt{2}$ times larger than the $\phi$ velocity, which arises directly from Equation (18) and accomplishes the Virial theorem.

The full steps of the solution of Equation (10) are given in Ref. [4]. This Reference too shows that the gravitational mechanism, created by the Keplerian velocity field of the HQS (Equation (6)) is perfectly symmetric with orbital motions. In fact, the Keplerian velocity field simulates the effects of a central field of gravitational accelerations, while the orbital motions simulate the outward centrifugal effects, exactly as conceived in Newtonian gravity. It also is shown that the Keplerian velocity field correctly predicts all the actually known experimental observations. It in particular causes very precisely the observed gravitational light lensing effect, due to the differentiated refraction times by Equation (7b) for prograde and retrograde light path. Section 5.5 of Ref. 4 and Section 8. A2 of Ref. 16 show the details. However, some other effects too are predicted, for which actually there are no experimental data to confront withy. 


\section{Concluding Comments and Considerations.}

According to the STR, the velocity of light has the same value in any inertial reference and in a reference moving at a relative velocity $v$ with respect to the hypothetically stationary reference, the time axis has a different direction. It has a space like component $v$, so that, from the view of the stationary observer, its component along the time axis in his own reference is shorter and the rate of time evolution of all physical processes in the moving reference seems reduced to $t=t_{0}\left(1-v^{2} / c^{2}\right)^{-1 / 2}$. In their turn, the space-like distances, in the moving reference, have a time-like component and seem to be shorter. However, an observer in the moving reference can say the same about the times and distances in the hypothetically stationary reference, which is the problematic reciprocal symmetry problem of the STR. In the present scenario of the HQS, the velocity of light has a fixed value $c$ with respect to the local HQS. In a reference, moving at a velocity $V$, with respect to the local $H Q S$, the velocity of light is anisotropic and the time evolution of all the physical processes is lowered because of the effective velocities $\left(c^{2} \pm V^{2}\right)^{1 / 2}$. The time evolution is given by

$T=T_{0}\left(1-V^{2} / c^{2}\right)^{-n}$ where $n=1 / 2$ for transverse oscillations and $n=1$ for longitudinal oscillations. The spatial distances are not altered and the reciprocal symmetry does not exist.

According to GR, from the view of an external observer, the rate of the time evolution $t^{\prime}$ of the physical processes (clocks), in a laboratory stationary within a gravitational field, is lower as indeed observed, given by $t^{\prime}=t_{0}^{\prime}\left(1-2 G M / r c^{2}\right)^{-1 / 2}$, where $2 G M / r$ is the local escape velocity. GR explains this in terms of spacetime curvature, in which the time axes have different directions inside and outside the field. Within the field, it has a component along $r$. The problem with GR is that it cannot explain why the gravitational slowing by the solar field is absent on the GPS clocks, moving with earth round the sun. It cannot, because the orbital velocity of earth cannot cancel the space-like velocity component along $r$. It in fact adds a new component. In the scenario of the present HQS dynamics gravitation, a body (clock), stationary in the ordinary space within a gravitational field, has an implicit velocity $V(r)=-(G M / r)^{1 / 2} \boldsymbol{e}_{\phi}$ with respect to the local HQS, due to the Keplerian velocity field of the HQS along $+\phi$, creating the gravitational field. This implicit velocity of the body is a true velocity with respect to the local moving HQS. For longitudinal oscillations of the clock's time standard, the clock rate is $T(r)=T_{0}(r)\left(1-G M / r c^{2}\right)^{-1}$, which causes exactly the same clock slowing as predicted by GR. In the language of GR, the time axis of this stationary clock has a space-like component along $-\phi$ (not along $r$ ). In this situation, the orbital velocity of earth along $+\phi$ naturally cancels the implicit velocity (and the space like component of the time axis) and thereby cancels the gravitational slowing of GPS clocks, due to the solar field. However, only orbital motions along direct circular equatorial orbits can cancel this implicit velocity. Any different orbital velocities do not cancel the gravitational slowing, as demonstrated by the GPS clocks in the non-equatorial orbits round earth. The orbit- 
al motion of earth gives in addition a natural explanation to the absence of light anisotropy with respect to earth; because the orbiting earth is stationary with respect to the local moving HQS.

Also, according to GR, from the view of an external observer, the velocity of a light pulse, propagating, toward the gravitational center, will seem to gradually reduce its velocity. GR explains this reduction in terms of a time like (implicit) component of the radial distances (stretching of the radial distances), due to spacetime curvature, which the external observer cannot see. From the view of the HQS dynamics gravitation the radial velocity component of light $c^{\prime}$ really decreases, given by $c^{\prime}=\left(c^{2}-G M / r\right)^{1 / 2}$. It decreases because the light pulse necessarily develops a lateral velocity component $(G M / r)^{1 / 2}$ along $-\phi$, which is due to the refraction rate (Equation (7a)) of the light velocity component along $r$. This component too is not seen by the external observer.

From the view of the present HQS dynamics, the absence of the gravitational slowing of the GPS clocks and the absence of light anisotropy with respect to earth are both due to the fact that earth is very closely stationary with respect to the local HQS in the Keplerian velocity field creating the solar gravitational field. Moreover, all the experimental observations with particles, having very high velocities within the earth-based laboratories that apparently corroborate the predictions of the TR, do not corroborate them at all. These observations simply are the effects of the very high velocities with respect to the local very slowly moving HQS. All these observations are the obvious signature of the ingenious outside-inside centrifuge mechanism of gravity, created by the solar Keplerian velocity field of the HQS.

The concern of the present work has not been simply constructing mathematical models that can simulate the observations, however to get understanding for these observations in terms of real and genuine physical mechanisms. This has largely been achieved. The HQS, retrieves locally an absolute (however non-universal) reference for rest and for motions, recovering locally an intrinsic meaning for motions. In the present view, the local HQS plays a fundamental role in the microscopic world of quantum physics as well as in the macroscopic world of gravitation, opening a way toward the unification of the fundamental forces.

\section{Conflicts of Interest}

The author declares no conflicts of interest regarding the publication of this paper.

\section{References}

[1] Einstein, A. (1905) Annalen der Physik, 17, 891-921.

https://doi.org/10.1002/andp.19053221004

[2] Lorentz, H.A., Einstein, A., Minkowski, H. and Weyl, H. (1923) The Principle of Relativity. Dover Publications, New York.

[3] Hatch, R.R. (2007) Physics Essays, 20, 83-100. https://doi.org/10.4006/1.3073811 
[4] Schaf, J. (2018) Journal of Modern Physics, 9, 111. https://doi.org/10.4236/jmp.2018.95068

[5] Schaf, J. (2018) Journal of Modern Physics, 9, 395. https://doi.org/10.4236/jmp.2018.93028

[6] Laue, M.V. (1955) Annalen der Physik, 38.

[7] Schwarzschild, K. (1916) Sitz. Preuss. Akad. d. Wiss., Part 1, 424.

[8] Ashby, N. (1996) Mercury, 23-27.

[9] Higgs, P.W. (1964) Physical Review Letters, 13, 508. https://doi.org/10.1103/PhysRevLett.13.508

[10] Englert, F. and Brout, R. (1964) Physical Review Letters, 13, 321. https://doi.org/10.1103/PhysRevLett.13.321

[11] Anderson, P.W. (1963) Physical Review Journals Archive, 130, 439. https://doi.org/10.1103/PhysRev.130.439

[12] Ginzburg, V.L. and Landau, L.D. (1950) Journal of Experimental and Theoretical Physics (JETP), 20, 1064.

[13] Meissner, W. and Ochsenfeld, R. (1933) Naturwissenschaften, 21, 787-788. https://doi.org/10.1007/BF01504252

[14] Carrol, S.M. (2000) The Cosmological Constant. arXiv:astro-ph/0004075

[15] Sola, J. (2013) Cosmological Constant and Vacuum Energy: Old and New Ideas. arXiv:1306.1527v3 [gr-qc]

[16] Schaf, J. (2019) JMP, 10, 225-255. https://doi.org/10.4236/jmp.2019.103018

[17] Dias, F.T., et al. (2010) Physica C: Superconductivity and Its Applications, 470, S111-S112. https://doi.org/10.1016/j.physc.2010.01.018

[18] Ives, H.E. and Stilwell, G.R. (1938) Journal of the Optical Society of America, 28, 215-226. https://doi.org/10.1364/JOSA.28.000215

[19] Hatch, R.R. (2004) GPS Solutions, 8, 67-73. https://doi.org/10.1007/s10291-004-0092-8

[20] Hatch, R.R. (2004) Foundations of Physics, 34, 1725-1739. https://doi.org/10.1007/s10701-004-1313-2

[21] Shapiro, I.I., et al. (1971) Physical Review Letters, 26, 1132. https://doi.org/10.1103/PhysRevLett.26.1132

[22] Miller, D.C. (1933) Review of Modern Physics, 5, 203. https://doi.org/10.1103/RevModPhys.5.203

[23] Pound, R.V. and Snider, J.L. (1965) Physical Review Journals Archive, 140, B788. https://doi.org/10.1103/PhysRev.140.B788

[24] Schaf, J. (2018) Journal of Modern Physics, 9, 2125. https://doi.org/10.4236/jmp.2018.912133 